\title{
PSYCHOLOGICAL SKILLS RELATED TO INTERCULTURAL ADJUSTMENT
}

\author{
David Matsumoto ${ }^{1}$, Satoko Hirayama ${ }^{2}$, Jeffery A. LeRoux ${ }^{3}$
}

\section{INTRODUCTION}

People from all corners of the globe migrate or sojourn to different cultures to work, study, and live, and in doing so face many issues in dealing with and adjusting to a new culture. Research has well documented the stresses, trials, and tribulations of sojourners and immigrants, and has identified the important affective, cognitive, and behavioral skills, abilities, and aptitudes that contribute to successful, and unsuccessful, sojourns. Despite this wealth of knowledge, however, the field has been relatively slow in identifying the core psychological components associated with positive intercultural adjustment. In this chapter, we report work from our laboratory spanning 17 studies on the development and validation of a new measure of intercultural adjustment - the Intercultural Adjustment Potential Scale (ICAPS) - that has helped us identify the psychological skills related to intercultural adjustment for highly functioning people around the world. The goal of this chapter is to introduce the reader to those skills. We begin by discussing the importance of intercultural adaptation and adjustment, and then review the literature identifying the factors that influence adjustment. We then discuss our theoretical approach to understanding adjustment, and describe the development and validation of our ICAPS measure. Finally we highlight the psychological skills that multiple studies have demonstrated are correlated with adjustment.

\section{INTERCULTURAL ADAPTATION AND ADJUSTMENT}

\subsection{Comparing and Contrasting Adaptation and Adjustment}

In our work we have found that it is important to make a distinction between adaptation and adjustment. Using Ward's approach, we believe that adaptation is based in the sociocultural domain (Ward, 2001), that is, it is the process of altering one's behavior to

${ }^{1}$ San Francisco State University

${ }^{2}$ Ochanomizu University

${ }^{3}$ San Francisco State University 
fit in with a changed environment or circumstances, or as a response to social pressure. One of the most well known models of adaptation, for instance, is Berry's (1994) analysis of the interaction styles for sojourners, immigrants, and refugees. In this model, four categories of interaction style are identified on the basis of the yes/no answers to two questions: (1) is it important to maintain my cultural identity and characteristics, and (2) do I value and want to maintain relationships with people of the host culture? Individuals who say yes to both are considered "integrators;" those who say no to both are "marginalizers." Those who say yes to the first and no to the second are "separators," while those who say no to the first and yes to the second are "assimilators." clearly these processes refer to behavioral changes made in response to different environments.

In contrast, we define adjustment as the subjective experiences that are associated with and result from attempts at adaptation, and that also motivate further adaptation. Adjustment, therefore, essentially refers to the general concept of well-being, which is an affective evaluation of one's life situation. In line with this definition, previous researchers have incorporated a wide range of outcome measures of adjustment, including self-awareness and self-esteem (Kamal \& Maruyama, 1990), mood states (Stone Feinstein \& Ward, 1990), and health status (Babiker, Cox, \& Miller, 1980) (all cited in Ward, 2001). Other typical measures of adjustment involve other subjective experiential constructs that refer to well-being, such as anxiety, mood, depression, subjective well-being, satisfaction, and happiness.

Some writers have developed synthesizing strategies to integrate these specific approaches to the definitions of adaptation and adjustment in order to highlight a smaller number of features. For example, Brislin (1981) has identified three factors of adjustment, including (1) having successful relationships with people from other cultures; (2) feeling that interactions are warm, cordial, respectful, and cooperative; and (3) accomplishing tasks in an effective and efficient manner. Hammer, Gudykunst, and Wiseman (1978) focused on these factors, and also included the ability to manage psychological stress effectively. Black and Stephens (1989) identified general adjustment involving daily activities, interaction adjustment involving interpersonal relations, and work adjustment related to work and tasks.

Research on adjustment outcomes for various adaptation strategies further highlights the conceptual difference between the two concepts. For example, while it is often politically correct to suggest that Berry's strategy of integration produces the best adjustment outcomes, in fact that is not often the case (see reviews by Berry \& Sam, 1997; Ward, 2001; Rudmin, 2003a, 2003b). It appears that people who engage in separation or assimilation adjust just as well to their life contexts as do people who integrate. Research is not as clear about marginalization. We would propose that marginalizers often feel well-adjusted, but that the people around them probably do not feel that they are.

\subsection{Positive and Negative Adjustment}

As alluded to above, adapting to a new culture can have both positive and negative adjustment outcomes. On one hand the positive consequences include gains in language competence; self-esteem, awareness, and health (Babiker et al., 1980; Kamal \& Maruyama, 1990); self-confidence, positive mood, interpersonal relationships, and stress reduction (Matsumoto et al., 2001). Clearly when intercultural experiences go well, many individuals 
report evolving in many qualitative, positive ways so that they are fundamentally different, and better, individuals. These include the development of multicultural identities and multiple perspectives with which to engage the world.

On the other hand, the negative consequences include psychological and psychosomatic concerns (Shin \& Abell, 1999); early return to one's home country (Montagliani \& Giacalone, 1998); emotional distress (Furukawa \& Shibayama, 1994); dysfunctional communication (Gao \& Gudykunst, 1991; Okazaki-Luff, 1991); culture shock (Pederson, 1995); depression, anxiety, diminished school and work performance, and difficulties in interpersonal relationships (Matsumoto et al., 2001). In extreme cases negative adjustment results in antisocial behavior (gangs, substance abuse, crime) and even suicide. Fortunately all sojourners do not experience this wide range of psychological and physical health problems, but most have probably experienced some of these problems at some point in their sojourn.

One of the goals, therefore, of intercultural adaptation is to adopt an adaptation pattern that minimizes these stresses and negative adjustment outcomes, and maximizes positive ones.

\section{FACTORS THAT PREDICT ADJUSTMENT}

\subsection{Previous Literature}

There have been many attempts to identify the factors that influence intercultural adjustment (reviewed in Matsumoto, 1999; Matsumoto et al., 2001). Studies have identified a wide range of variables, such as knowledge, language proficiency, attitudes, previous experiences, levels of ethnocentrism, social support, cultural similarity, adventure, and self-construals. Among these, three factors have consistently emerged as leading contributors to adjustment: knowledge of host and home culture, ethnocentrism, and language proficiency. In fact it is precisely because of these factors that many intercultural training interventions involve language skill and knowledge training. The underlying assumption of such training is that if people can speak the language of the host culture, and if they know some basic facts about the host culture, they can adjust to life better. Likewise, if people can recognize the existence of ethnocentrism - how our own cultural upbringing contributed to how we interact with the world and with others - and can recognize that our viewpoint is one of many valid and legitimate views, they will have successful adjustments.

\subsection{A New Way of Understanding Intercultural Adjustment Focused on Emotion}

In our work, we have chosen to focus on the social psychology of adjustment through an analysis of intercultural encounters. Because of differences in language, nonverbal behaviors, values, norms, attitudes, rules, systems, and other manifestations of culture, intercultural adjustment is replete with conflict, frustration, and struggle. They are, in fact, inevitable because of cultural differences, at least on the level of manifest behaviors (despite possible cultural similarities in underlying psychological goals and intentions). 
These conflict-arousing contexts are seeped with emotion, often negative. Once aroused, intercultural encounters can easily lead to negative experiences, frustrations, stereotypes, attitudes, and a host of other affective/cognitive outcomes that are not conducive to successful adaptation. Therefore, we believe one of the keys to successful intercultural adjustment is the ability to regulate one's emotions, and to not allow negative affect, which may be inevitable, to overcome oneself and color one's cognitions and motivations.

Our theoretical framework was informed by the literature on stress and coping. While this literature of course indicates that major life events - marriage, divorce, births and deaths - have consequences on well-being, research has also emphasized the cumulative effects of day-to-day events, both positive and negative, on well-being and relationships (DeLongis, Folkman, \& Lazarus, 1988; Kanner, Coyne, Schaefer, \& Lazarus, 1981). This literature has shown that daily hassles and uplifts can have major effects on people's physical and psychological health, that is, adjustment, that is similar to or even stronger than the effects of major life events. This notion is even more relevant in the intercultural context, where daily life is replete with daily hassles because of the new cultural context in which to adapt and adjust, the inherent ambiguity of new cultural systems and intercultural communication, and the inevitable conflict that arises because of differences. Emotions, therefore, play a major role in adaptation and adjustment, just as they play a major role in the stress and coping process.

Emotions, in fact, are a large part of our lives. Emotions color our experiences, giving them meaning and relevance for our well being. Sadness, anger, disgust, fear, frustration, shame, and guilt - while all negative and often unattractive to us, are all important in that when we feel these emotions, they tell us something important about ourselves and our relationships with other people, events, or situations. Happiness, joy, satisfaction, pleasure, and interest are also important emotions, in that they, too, give us important information about our relationships with others. Emotions are "read-out mechanisms" because they provide information to us about our status in relation to the world (Buck, 1984).

Emotions are also important because they motivate behaviors. Sadness and anger, for example, make us do something, just as happiness and joy reinforce behaviors. The father of modern day research and theory of emotion in psychology - Sylvan Tomkins - suggested that emotions are motivation and if you want to understand why people behave the way they do, you had to understand their emotions (Tomkins, 1962, 1963). For these reasons, it is only natural that we give more consideration to this most-important and deserving part of our lives. We thus turned our attention to developing a theoretical framework that incorporated emotion and its regulation in explaining and predicting intercultural adjustment.

Emotion regulation allows individuals to engage in clear thinking about intercultural incidents without retreating into psychological defenses. If sojourners do not have the ability to regulate or control their emotions, they will be unlikely to adjust well because they will be locked into their automatic or habitual ways of thinking and interacting with the world. Emotion regulation seems likely to be the gatekeeper skill for intercultural adjustment.

The ability to regulate emotions, however, alone is not sufficient. Once held in check, individuals must then engage in learning about the new culture, which requires them to analyze the cultural underpinnings of the context, and understand the intentions and behaviors that produced conflict in the first place from a different cultural perspective. 
In doing so, individuals may draw on the reservoirs of knowledge that previous research has shown to be important in predicting adjustment, such as knowledge of host and own culture, previous experience, and the like. Important aspects of this ability to learn a new culture are likely to include being free of over-attachment to previous ways of thinking that have worked in the past and a willingness to tolerate the ambiguity of not knowing or being able to predict the likely outcomes of one's actions. One also needs the openness and flexibility to call to mind and to consider alternatives that would have been inappropriate in previous social experience. A further necessity in adjusting to a new culture must be the monitoring of the behaviors and reactions of one's self and others around one's self. It may also be the case that conscious, critical thinking about intercultural incidents, the generation of rival hypotheses that explain conflict other than those from their own cultural framework and the creation of a new set of ideas about social interaction are critical to adjustment.

This model is inherently a growth model. By engaging in critical thinking about cultural differences and being open and flexible to new ways of thinking, one allows for one's ways of person perception to grow. One continually adds more complexity to one's method of interacting with diversity. All of this is possible, however, only when emotions are regulated and negative emotions do not get the best of oneself.

If, however, negative emotional reactions overwhelm, people cannot engage in critical thinking about those differences. Rather, they regress to a previous way of thinking that is rooted in ethnocentrism and stereotype. Instead of creating rival hypotheses that will stimulate growth in thinking, people instead only reinforce pre-existing, limited thinking. Openness and flexibility to new ideas is not even an option because these new ideas don't exist. Instead, there is only a regurgitation of stereotypes and ethnocentric attitudes. This is a non-growth model.

The four main ingredients to personal growth in relation to dealing with cultural differences, therefore, is emotion regulation, critical thinking, openness, and flexibility. Of these, emotion regulation is the gatekeeper of the growth process, because if one cannot put inevitable negative emotions in check, it is impossible to engage in what is clearly higher order thinking about cultural differences.

Our approach to understanding intercultural adjustment is based on a stress and coping approach, which appears to offer the broadest framework to understand this issue. According to Ward (2001), the factors that predict intercultural adjustment include life changes, cognitive appraisals of stress, coping styles, personality, social support, cultural identity, type of acculturating groups, perceived discrimination, acculturation strategies, and sociocultural adaptation. Because intercultural contact and change are significant life events that are inherently stressful, coping skills are required to deal with life in a new and different environment (Ward, 2001). Models of cross-cultural coping such as problem focused vs. emotion focused, engagement vs. avoidance, and primary vs. secondary control have provided ample evidence to predict adjustment.

To be sure, these psychological skills - emotion regulation, openness, flexibility, and critical thinking - have been described in the previous literature. To our knowledge, however, they have not been empirically compared nor integrated into a single set of dimensions that accounts for intercultural adjustment. The studies we describe below represent an attempt to create a more complete model of the psychology of intercultural adjustment. 


\section{THE ASSESSMENT OF PSYCHOLOGICAL SKILLS THEORETICALLY RELATED TO INTERCULTURAL ADJUSTMENT - THE INTERCULTURAL ADJUSTMENT POTENTIAL SCALE (ICAPS)}

\subsection{Development of the ICAPS}

For years the field has struggled with the creation of valid and reliable individual difference measures that will predict intercultural adjustment, for various reasons. For one, when researchers focus on variables that are culture- or context-specific, such as knowledge of host culture, it is nearly impossible to generate a valid measure because it would necessitate the assessment of knowledge that is specific to different cultures, and would be based on the assumption that such knowledge existed and was applicable to all contexts within that culture. Such measures would border on the assessment of knowledge of cultural stereotypes.

Another factor that has hindered the development of valid and reliable individual difference measures has been a lack of consensus on the underlying psychological components that are important to adaptation. As mentioned above, while previous research has identified many variables that appear to be associated with successful adjustment, there is no coherent model that unifies these components into a comprehensive and understandable framework for predicting adaptation. The identification of several psychological variables as the keys to intercultural adaptation bypasses the problem of culture-specific knowledge, and views the potential for intercultural adjustment as a function of the psychological skills that individuals possess within them. In doing so, it does not rely on the knowledge or attitudes that sojourners or immigrants may have, or on the specific host culture in which they reside, or on language proficiency. Thus, the potential applicability of this approach is considerably larger than previous models that are tied to knowledge and attitudes.

Because there was no measure that could assess individual differences in the potential for intercultural adjustment based on the factors outlined above, we opted to create our own, resulting in the development of the Intercultural Adjustment Potential Scale ICAPS. Our strategy was to embody the several factors previously suggested as being related to intercultural adjustment in a pool of items and then to empirically test which had the strongest ability to predict intercultural adjustment, rather than to decide on an a priori basis which items should be included.

We thus examined item content from a number of valid and reliable personality inventories assessing psychological constructs related to emotion regulation, critical thinking, openness/flexibility, interpersonal security, emotional commitment to traditional ways of thinking, tolerance of ambiguity, and empathy. These included the Eysenck Personality Inventory (EPI), the Beck Depression Inventory (BDI), the State-Trait Anxiety Inventory (STAI), the Bem Sex Role Inventory (BSRI), the original Minnesota Multiphasic Personality Inventory (MMPI) item pool, the California Personality Inventory (CPI), the NEO Personality Inventory (NEO-PI), the Big Five Personality Inventory (BFI), the California F-Scale, and the Interpersonal Reactivity Index. We created items based on the ideas gleaned from our examination of these scales, and also constructed our own items. This resulted in the initial development of 193 items.

One issue that arose early in this work was whether this test would be developed for any sojourner of any cultural background, or for those from a single culture. We opted for the 
latter, assuming that it would be more beneficial to create and validate a measure that has as high a predictive validity as possible for one cultural group, rather than develop a general measure at the sacrifice of predictive validity. The development of a culture-general measure would require the testing of people from multiple home cultures in multiple host cultures, which would be practically infeasible. If a culture-specific measure were created, however, it could serve as the baseline platform for similar method development in other cultures. Thus, we focused on Japanese sojourners and immigrants, because of the literature in the area and our own expertise with this culture.

Because we were concerned with the cross-cultural equivalence of the 193 items, to take into account that respondents might have different English language capabilities, and to remove any colloquialism and difficulty of wording, two researchers created the items, reviewing and modifying all items in terms of language and style, rendering the wording appropriate for Japanese students who might possess a limited selection of English idioms commonly in use. Two native Japanese research assistants then reviewed the new items, ensuring that they were understandable to native Japanese. Care was taken to exclude items that depended for their utility on a cultural value in which Japanese and U. S. culture differ. In all cases, items were written to adapt the cultural meaning of an item in the United States to the same cultural context from a Japanese perspective.

The final selection of items was based on their empirical ability to predict intercultural adjustment, rather than their theoretical potential for prediction. Items having little or nothing to do with intercultural adjustment were eliminated, even if elsewhere they reliably measured an aspect of an underlying psychological skill (e.g., openness) that was theoretically purported to be related to adjustment. Also, some items predicted adjustment better than others; thus, only items that predicted adjustment the best, according to empirical criteria, were retained. This resulted in a final pool of 55 items. To date we have conducted 17 studies documenting the reliability and validity of this 55-item ICAPS in predicting intercultural adjustment success. Table 1 shows a summary of the findings. The evidence demonstrates the unique contribution of the ICAPS to the field.

\subsection{Validity Studies of the ICAPS}

The first eight studies provided considerable evidence for the internal, temporal, and parallel forms reliability of the ICAPS-55; for its predictive ability with not only subjective indices of adjustment, but also using psychometrically standardized measures, peer ratings, and expert ratings based on interviews with the participants; for its convergent validity with a similar measure; for its construct validity with various personality scales; for its incremental validity; and for its external validity in predicting changes as a result of intercultural seminars, and in identifying experts who work in the intercultural field (Matsumoto et al., 2001).

Encouraged by our initial studies, we engaged in further testing of the ICAPS, interested in the boundaries of its predictive abilities (Matsumoto et al., 2003; Matsumoto, LeRoux, Bernhard, \& Gray, 2004). In Studies 9 through 14, we were particularly interested in ascertaining whether the ICAPS could predict adjustment in Japanese non-student samples and for non-Japanese. The results of these studies replicated and considerably extended the findings reported previously by Matsumoto et al. (2001). ICAPS scores predicted adjustment in Japanese non-student samples in a variety of ways, including 


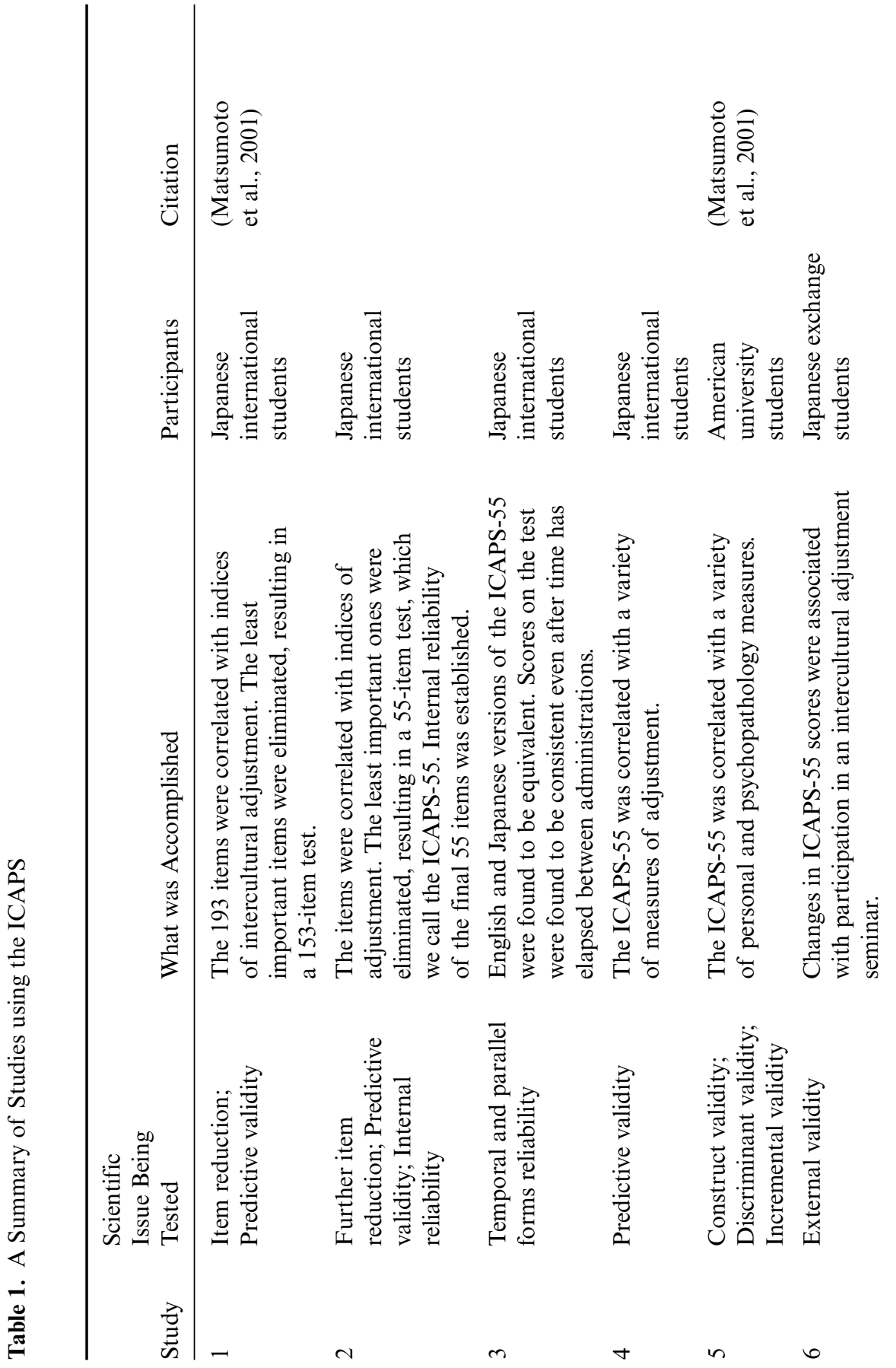



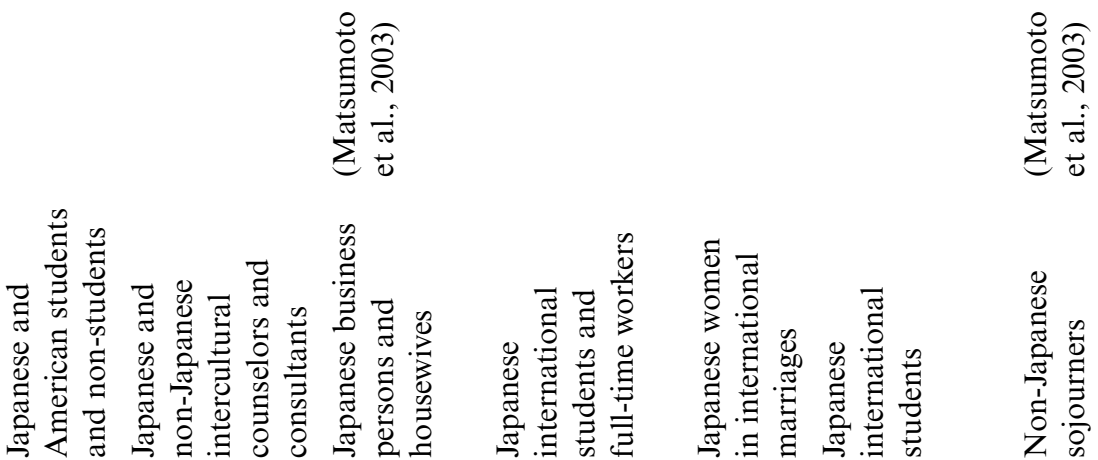

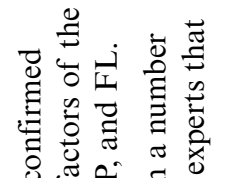

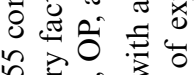

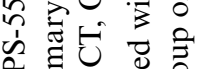

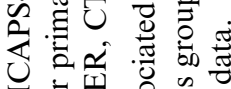

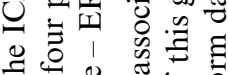

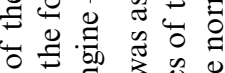

के

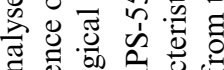

च ए

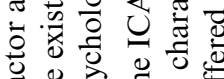

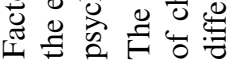
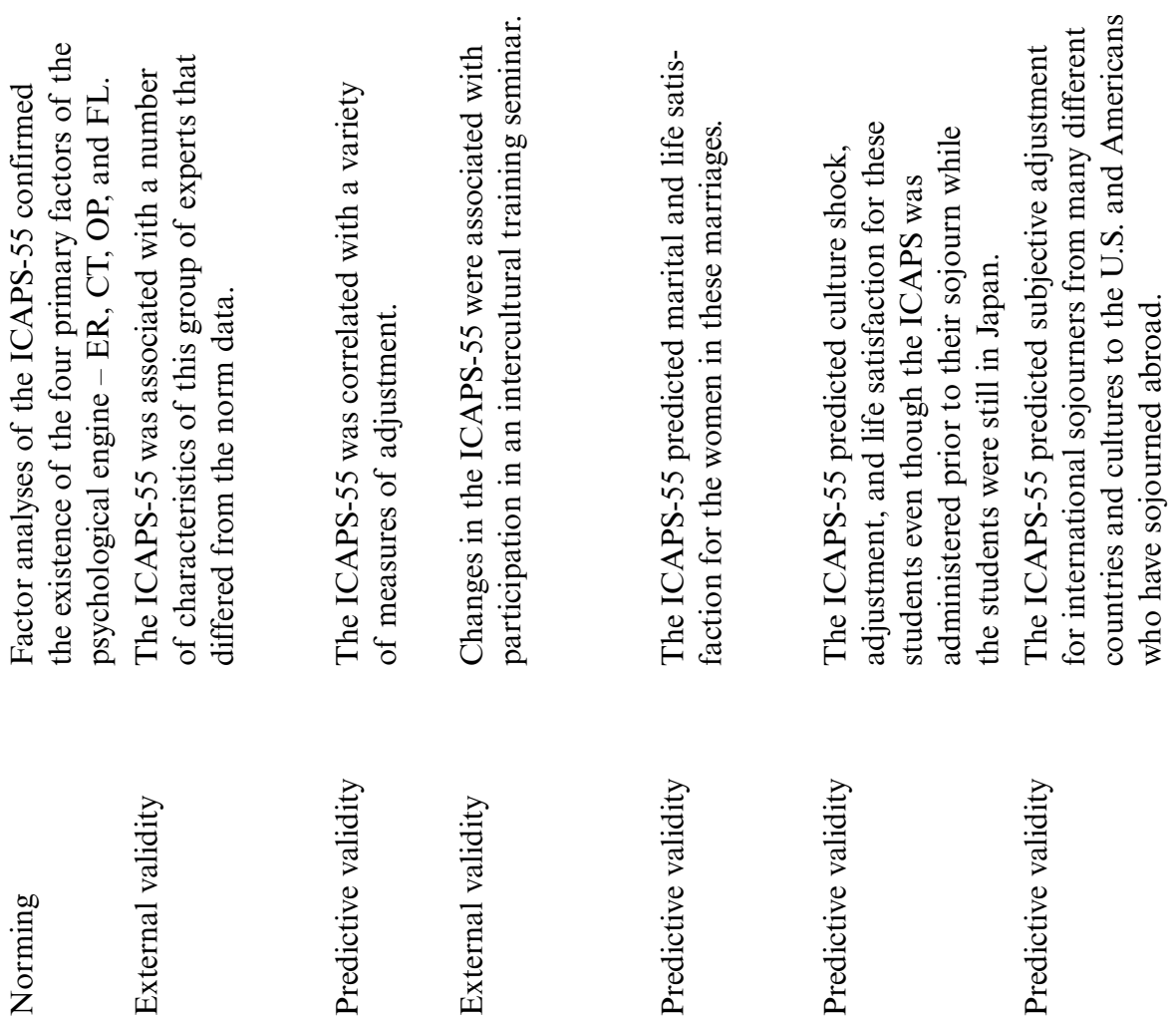

:

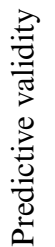

$?$
0
0
0
0
0
0
0
0
0
0
0

$\wedge$

$\infty$

$a$

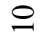

$\exists$

$\simeq$

$\underline{2}$

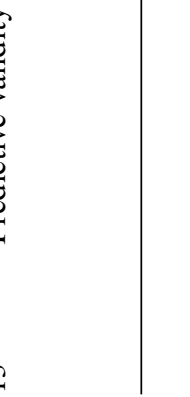




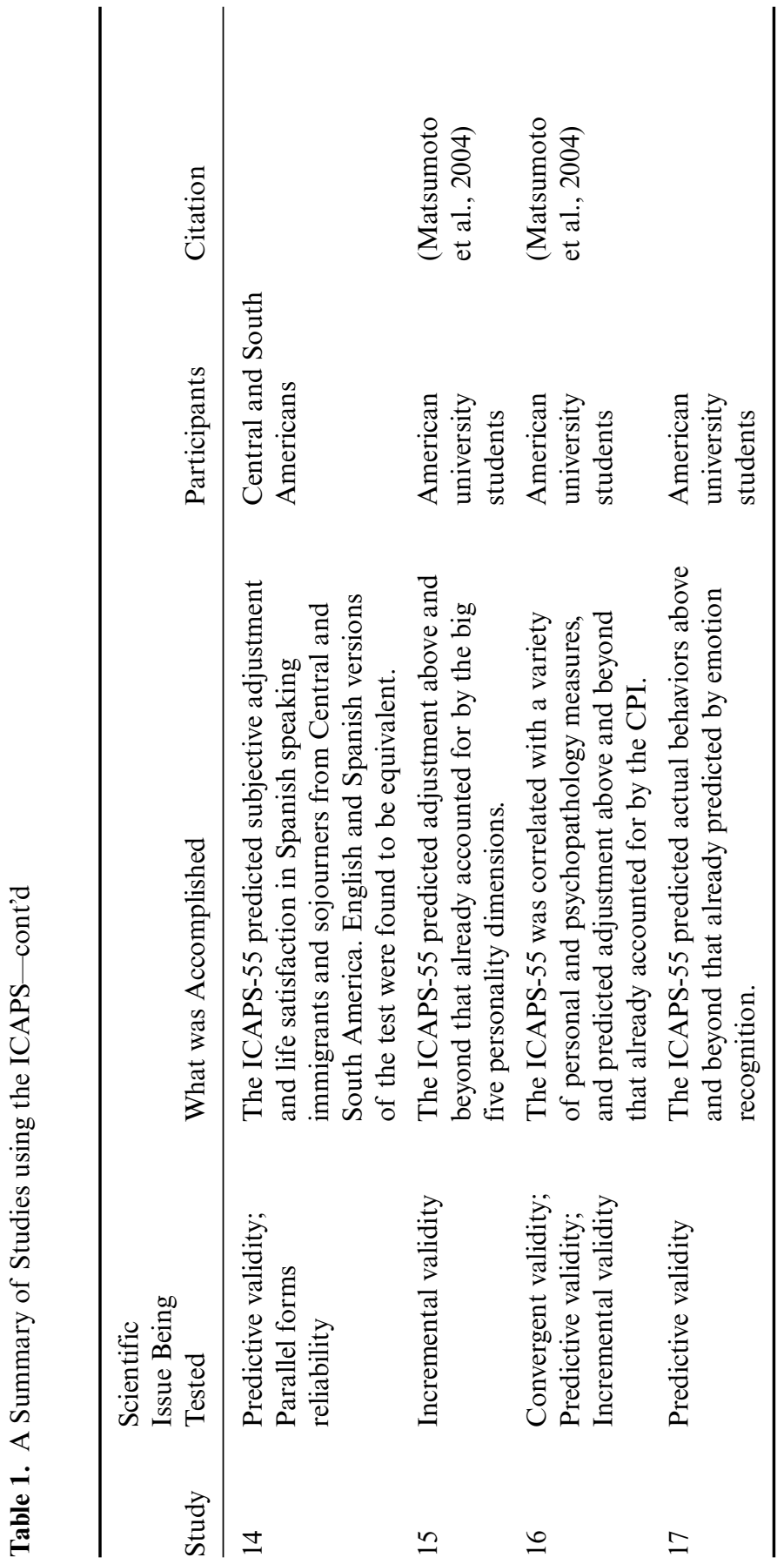


standardized measures of anxiety and depression, subjective indices of adjustment, satisfaction with life, and marital satisfaction. The ICAPS also predicted culture shock in Japanese student sojourners even when ICAPS was administered prior to these individual's leaving Japan, and subjective adjustment in a general immigrant sample, as well as in samples from India, Sweden, and Central and South America. Finally, the ICAPS predicted subjective adjustment and satisfaction with life in Americans who had sojourned abroad as well. Coupled with the eight studies described earlier, these findings lent strong and consistent support to the psychometric reliability and validity of the ICAPS to predict intercultural adjustment.

Most recently three additional studies (Studies 15 through 17) examined the personality and behavioral correlates of the ICAPS (Matsumoto et al., 2004). The findings from the studies provided strong evidence for the validity of the ICAPS scales to predict adjustment above and beyond that already predicted by personality. Furthermore, the ICAPS predicted actual behaviors above and beyond that already predicted by emotion recognition.

\section{THE PSYCHOLOGICAL SKILLS UNDERLYING THE ICAPS}

\subsection{Validity with Adjustment}

The ICAPS was originally validated using a total score summed across all 55 items. Factor analyses using normative data (n approximately 2,300, half of whom are non-U.S. born and raised) suggested that four factors underlay the ICAPS - Emotion Regulation (ER), Openness (OP), Flexibility (FL), and Critical Thinking (CT) (Matsumoto et al., 2001). These findings provided support for our theoretical formulation in which the importance of ER, OP, CT, and FL are the key psychological ingredients to intercultural adaptation. These skills were hypothesized as necessary in allowing immigrants and sojourners to cope with stress and conflict that are inevitable in intercultural sojourns, while at the same time allowing for personal growth in understanding, tolerance, and acceptance of cultural differences.

To obtain further support for the validity of these four psychological skills to predict adjustment, we created scores for each of these scales and computed correlations between them and various adjustment variables across the studies conducted to determine which psychological constructs predicted adjustment. Table 2 shows a summary of the findings. The first column indicates the ICAPS scales that were significantly associated with the adjustment measures described in the second column.

The findings demonstrated that individuals who scored high on the ICAPS scales had less adjustment problems in work, house work, spare time, and family domains; less somatic, cognitive, and behavioral anxiety; less depression; greater subjective well-being in their adjustment to the US or another country; greater subjective adjustment; higher dyadic adjustments in international marriage; higher life satisfaction; less psychopathology; less culture shock and homesickness; higher language scores; better grades; more tendency to work; higher income; and many of the skills that are useful to managers in solving the complex problems of running a business. The correlations with the various adjustment outcomes provided strong support for this conglomeration of skills to predict adjustment. 
Table 2. Adjustment that are Predicted by the ICAPS Scales

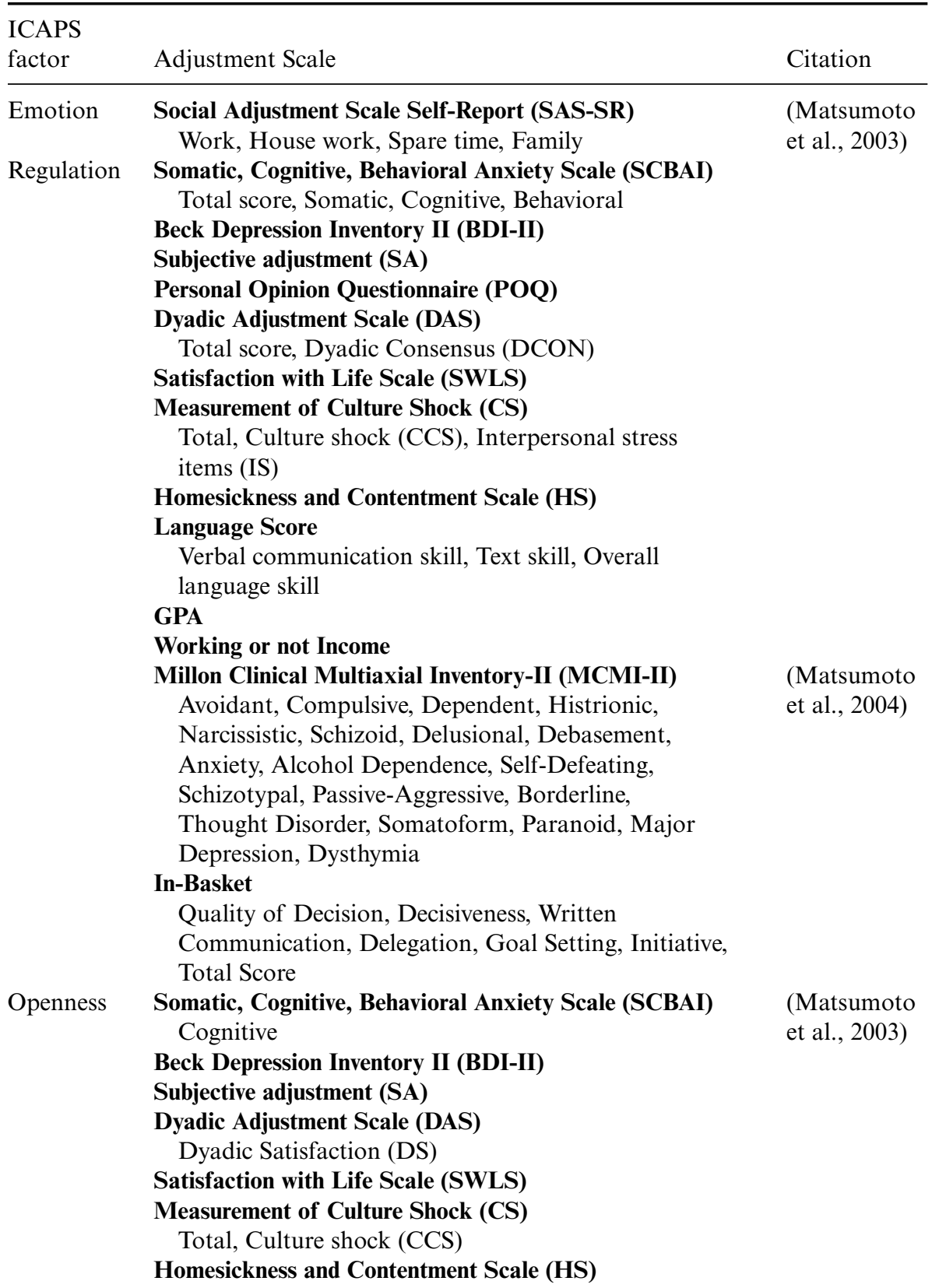


Table 2. Adjustment that are Predicted by the ICAPS Scales - cont'd

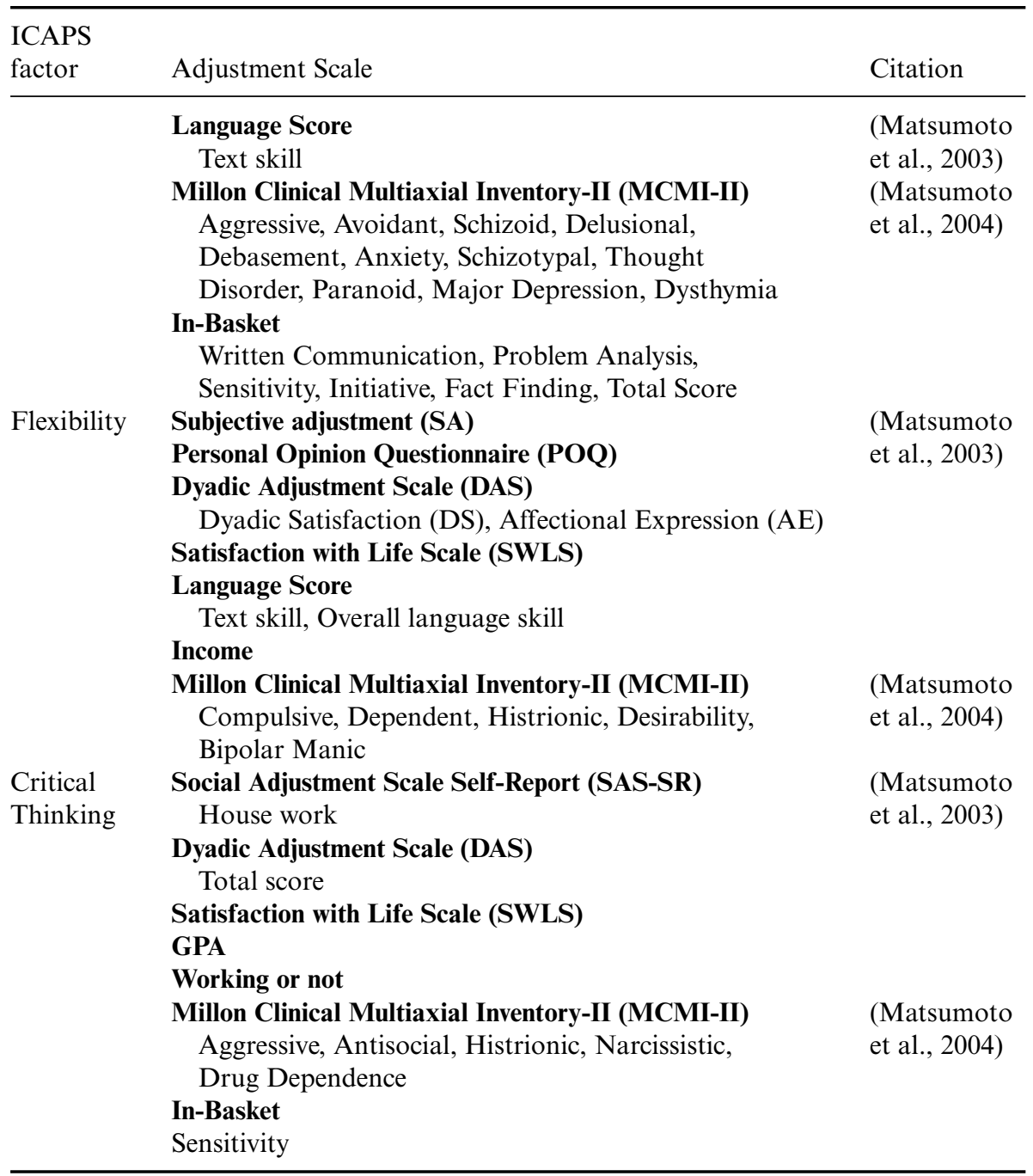


Conceptually we suggested that ER was a gatekeeper skill because it is necessary for people to manage inevitable intercultural conflict and that once emotions were regulated individuals could engage in critical thinking and assimilation of new cognitive schemas that aid in adjustment. Various outcomes across all studies supported this contention because, as shown in Table 2, ER predicted most of the adjustment measures relative to the other ICAPS scales. In addition, hierarchical multiple regressions indicated that ER accounted for most of the variance in various adjustment outcomes when entered first in the regression; the additional variance accounted for by OP, FL, and CT was always negligible (Matsumoto et al., 2003).

At the same time the strong findings for ER do not argue against the importance of OP, FL, and CT. Theoretically these skills make sense in understanding intercultural adjustment. Thinking out of the box and examining rival hypotheses to understand uncommon behavior, which is necessary in intercultural adjustment, cannot occur without CT. Assimilation of new cognitive schemas of the world cannot occur without OP and FL. That the empirical relationships of these factors to adjustment indices were not as strong as that for ER may be related to their factor order and lower proportion of variance accounted for relative to ER. Despite this fact these scales still emerged with significant betas in a number of the regression analyses including ER.

\subsection{Personality Traits Associated with Intercultural Adjustment Potential}

In our previous studies we also examined correlations between each of the ICAPS scores with personality dimensions (summarized in Table 3). The ICAPS scores converge with a number of personality dimensions. For example, correlations with the personality traits of the Five Factor Model (Matsumoto et al., 2004) indicated that ER was correlated with Neuroticism, which measures a person's inability to regulate emotion in a normal fashion (Costa \& McCrae, 1992). OP was correlated with Openness because it was constructed to be similar to Openness as measured in tests of the big five dimensions of personality. FL was negatively correlated with Conscientiousness because persons in the high ranges of Conscientiousness are characterized by rigidity in behavior and thought.

The ICAPS scores were also correlated with other personality traits. ER was correlated with the social ascendancy scales of the CPI as those are indicators of positive social skills and abilities. ER was also correlated with CPI Realization as it is associated with success in life, and negatively with Internality because it measures a tendency to withdraw from active involvement with the social world. ER was also correlated with the normative behavior scales of the CPI as most of these scales are indicative of internal organization, which leads to successful coping with one's culture of origin. ER and CT were associated with CPI scales measuring achievement and ability as these are aspects of successful coping, and self application requiring self-discipline and the ability to think critically, which are aspects of what we believe those parts of ICAPS measure. Finally ER, OP, and CT were positively correlate with CPI Psychological Mindedness as it predicts the dispassionate ability to analyze social situations and the mental processes required to cope in general, control one's own emotions, an openness to the various attributes of self, others and unique situations, as well as a capacity to think critically. These correlations and those reported in Table 3 provide a fairly comprehensive picture of the personality skills related to intercultural adjustment. 
Table 3. Personality Dimensions that are associated with the ICAPS Scales

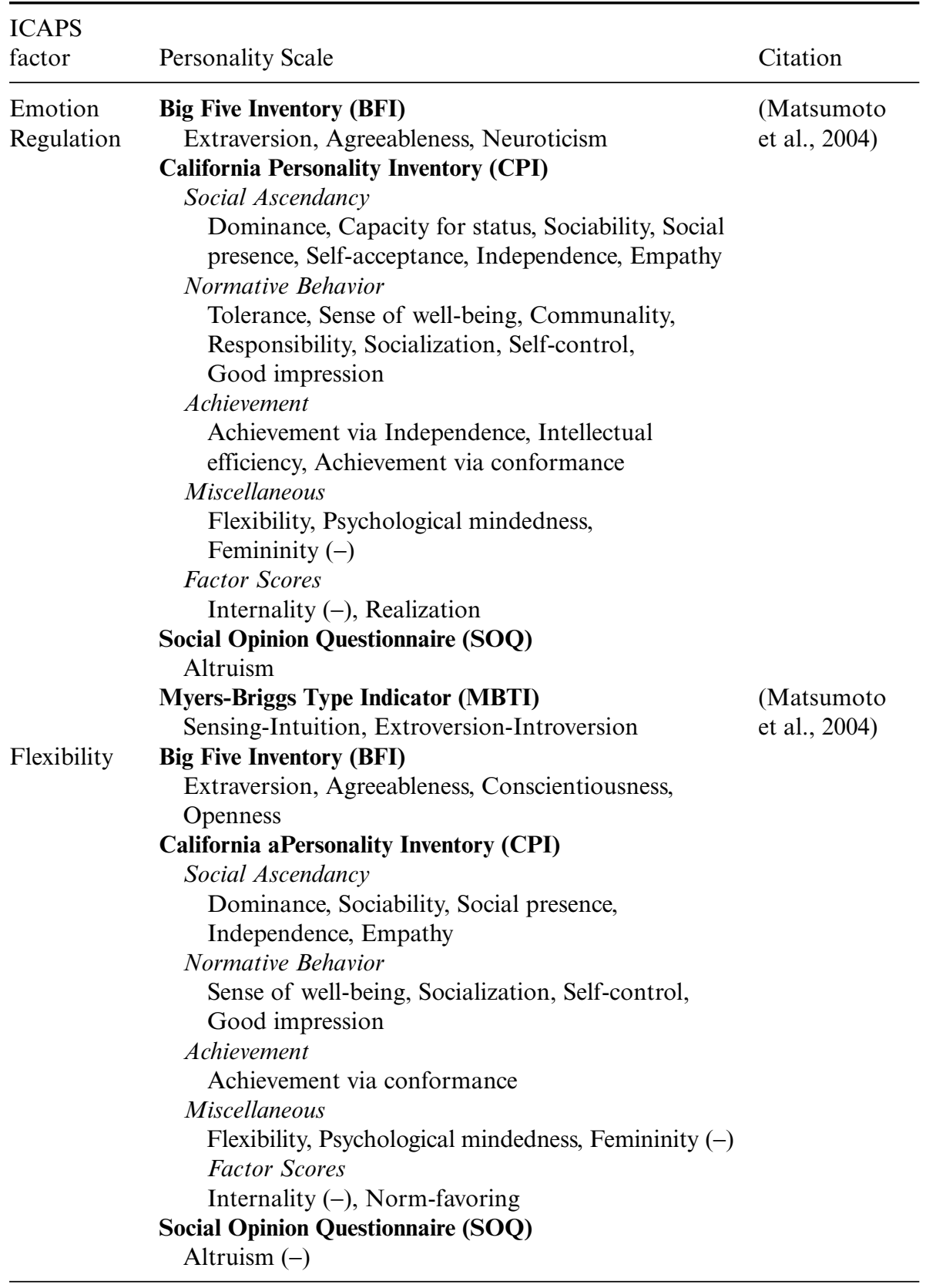


Table 3. Personality Dimensions that are associated with the ICAPS Scales - cont'd

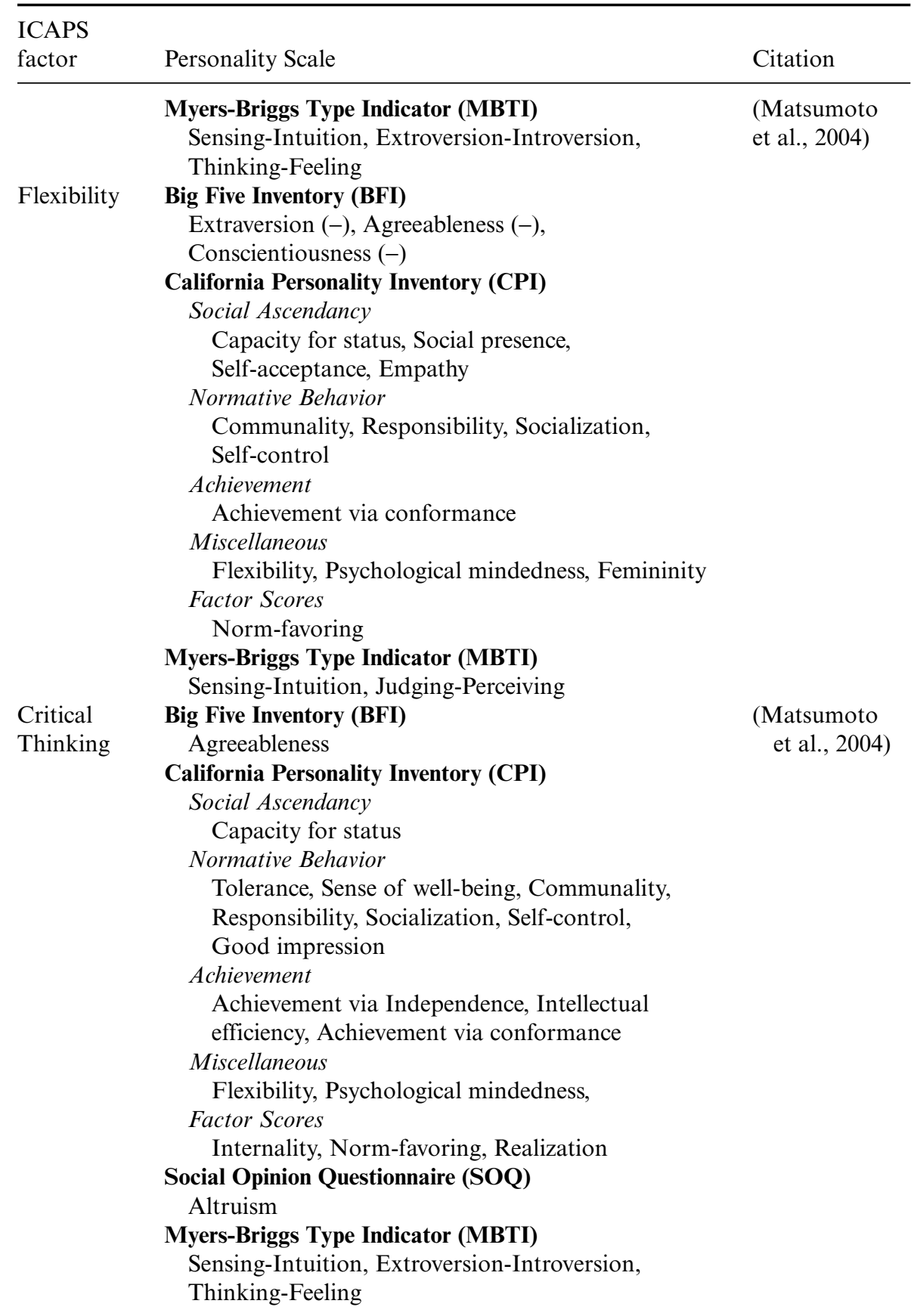

Note: (-) indicates negative correlation. 


\subsection{Studies Using the ICAPS by Others}

Findings documenting the validity of the ICAPS to predict adjustment have also been reported by other laboratories. For instance Savicki et al. (in press) investigated actual and potential intercultural adjustment using the ICAPS. His purpose was four fold: (1) To compare ICAPS and adjustment scores of study abroad students (SA) with those of students who stay at home (Home) at the beginning and the end of the study abroad semester; (2) To examine whether ICAPS scores of SA at pre-departure predict adjustment at the end of the foreign culture sojourn; (3) To examine whether ICAPS and personal adjustment scores of SA increase over their stay in a foreign culture; and (4) to examine whether ICAPS and personal adjustment are related to personality characteristics and preferred coping strategies.

A group of 19 students from an American university who studied abroad for three months (SA) were matched with 46 students who stayed in the U.S. during the same semester (Home). The groups were matched for age (19 to 25 , with $83 \%$ in the $20-22$ range), gender ( $54 \%$ women), and class standing (all juniors and seniors). They completed ICAPS, the Satisfaction with Life Scale (SWLS), a Five Factor Personality Questionnaire, the Life Orientation Test (LOT), and scales assessing Hope and Coping. SA students' voluntary participation was requested at four points in time: within one month prior to departure for their study abroad experience, during the beginning, middle, and end of the academic term of the study abroad experience.

The results indicated that the SA group was higher than Home students on ICAPS55, ER, and SWLS at the beginning of the semester, and on ER, CT, and SWLS at the end of the semester (Savicki, Downing-Burnette, Heller, Binder, \& Suntinger, in press). ICAPS-55 and ER predicted higher personal adjustment approximately four months later at the end of the academic term in the SA group (Savicki et al., in press). This finding supported the predictive validity of the ICAPS-55 score and the notion that ER may be a "gatekeeper" for intercultural adjustment potential (Matsumoto et al., 2001). As to the changes in the SA group, FL and SWLS increased at a statistically significant level between pre-departure and the end of the term (Savicki et al., in press).

Finally, a similar pattern of correlations appeared for the ICAPS-55 and ER with personality and coping variables (Savicki et al., in press). Those who had higher ICAPS55 and ER scores were outgoing, open to new experiences, positive in outlook, and hopeful of one's abilities and of potential outcomes. In addition, they applied specific strategies of dealing with stress that focused on actively planning and carrying out actions to directly reduce stress, as well as developing a positive framework for stressful situations. They were also less anxious, less negative in their outlook, less likely to ignore or withdraw from unpleasant events in the foreign culture. These findings were especially important because they were generated by researchers outside of our laboratory, and they, too, demonstrated the significance of the ICAPS in intercultural adjustment.

\section{CONCLUSION}

In this chapter, we reported recent work from our laboratory spanning 17 studies on the development and validation of a new measure of intercultural adjustment - the ICAPS. Focusing initially on sojourners from Japan, our studies suggested that the ICAPS 
is a highly valid and reliable measure to predict adjustment success for students, businesspersons, housewives, and individuals involved in intercultural marriages. Further tests indicated its validity for predicting adjustment in sojourners from other cultures as well. These findings in toto lead us to believe that the psychological constructs identified by the ICAPS are applicable to predicting intercultural success for sojourners from many different cultures. Individuals with the potential to adjust to life well in another culture are good at regulating emotions, open and flexible to new experience, and able to think critically. The availability of the ICAPS, and other measures like it, promises to continue to make a significant contribution to intercultural relations for years to come, and its findings point to the importance of certain key psychological skills important for adjustment.

\section{REFERENCES}

Babiker, I.E., Cox, J.L., \& Miller, P. (1980). The measurement of cultural distance and its relationship to medical consultations, symptomatology and examination of performance of overseas students at Edinburgh University. Social Psychiatry, 15, 109-116.

Berry, J. (1994). Acculturation and psychological adaptation. In A. M. Bouvy, F. J. R. v. d. Vijver, P. Boski \& P. Schmitz (Ed.), Journeys into cross-cultural psychology (pp.129-141). Lisse, Netherlands: Swets and Zeitlinger.

Berry, J., \& Sam, D. (1997). Acculturation and adaptation. In J. W. Berry, M. H. Segall \& C. Kagitcibasi (Ed.), Handbook of cross-cultural psychology, Vol 3: Social and behavioral applications. Boston, MA: Allyn and Bacon.

Black, J.S., \& Stephens, G.K. (1989). The influence of the spouse on American expatriate adjustment and intent to stay in Pacific Rim overseas assignments. Journal of Management, 15(529-544).

Brislin, R. (1981). Cross-cultural encounters. New York: Pergamon.

Buck, R.W. (1984). The communication of emotion. New York: Guilford Press.

Costa, P.T., \& McCrae, R.R. (1992). Revised Neo-Personality Inventory (NEO-PI-R) and Neo Five Factor Inventory (NEO-FFI). Odessa, FL: Psychological Assessment Resources.

DeLongis, A., Folkman, S., \& Lazarus, R. (1988). The impact of daily stress on health and mood: Psychological and social resources as mediators. Journal of Personality and Social Psychology, 54(3), 486-495.

Furukawa, T., \& Shibayama, T. (1994). Factors influencing adjustment of high school students in an international exchange program. Journal of Nervous and Mental Disease, 182(12), 709-714.

Gao, G., \& Gudykunst, W. (1991). Uncertainty, anxiety, and adaptation. International Journal of Intercultural Relations, 14(3), 301-317.

Hammer, M., Gudykunst, W.B., \& Wiseman, R.L. (1978). Dimensions of intercultural effectiveness: An exploratory study. International Journal of Intercultural Relations, 2, 382-393.

Kamal, A.A., \& Maruyama, G. (1990). Cross-cultural contact and attitudes of Qatari students in the United States. International Journal of Intercultural Relations, 14, 123-134.

Kanner, A. D., Coyne, J. C., Schaefer, C., \& Lazarus, R. (1981). Comparison of two modes of stress measurement: Daily hassles and uplifts versus major life events. Journal of Behavioral Medicine, 4, 1-39.

Matsumoto, D. (1999). Nihonjin no Kokusai Tekiouryoku. Tokyo: Hon no Tomosha.

Matsumoto, D., LeRoux, A.J., Bernhard, R., \& Gray, H. (2004). Personality and behavioral correlates of intercultural adjustment potential. International Journal of Intercultural Relations, 28(3-4), 281-309.

Matsumoto, D., LeRoux, A.J., Iwamoto, M., Choi, J., Rogers, D., Tatani, H., \& Uchida, H. (2003). The robustness of the intercultural adjustment potential scale (ICAPS): the search for a universal psychological engine of adjustment. International Journal of Intercultural Relations, 27, 543-562.

Matsumoto, D., LeRoux, J., Ratzlaff, C., Tatani, H., Uchida, H., Kim, C., \& Araki, S. (2001). Development and validation of a measure of intercultural adjustment potential in Japanese sojourners: The Intercultural Adjustment Potential Scale (ICAPS). International Journal of Intercultural Relations, 25, 483-510.

Montagliani, A., \& Giacalone, R.A. (1998). Impression management and cross-cultural adaptation. Journal of Social Psychology, 138(5), 598-608. 
Okazaki-Luff, K. (1991). On the adjustment of Japanese sojourners: Beliefs, contentions, and empirical findings. International Journal of Intercultural Relations, 15(1), 85-102.

Pederson, P. (1995). The five stages of culture shock: Critical incidents around the world. Westwood, CT: Greenwood Press.

Rudmin, F.W. (2003a). Critical history of the acculturation psychology of assimilation, separation, integration, and marginalization. Review of General Psychology, 7(1), 3-37.

Rudmin, F.W. (2003b). "Critical history of the acculturation psychology of assimilation, separation, integration, and marginalization": Correction to Rudmin (2003). Review of General Psychology, 7(3), 250.

Savicki, V., Downing-Burnette, R., Heller, L., Binder, F., \& Suntinger, W. (in press). Contrasts, changes, and correlates in actual and potential intercultural adjustment.

Shin, H., \& Abell, N. (1999). The homesickness and contentment scale: Developing a culturally sensitive measure of adjustment for Asians. Research on Social Work Practice, 9(1), 45-60.

Stone Feinstein, E., \& Ward, C. (1990). Loneliness and psychological adjustment of sojourners: New perspectives on culture shock. In D. M. Keats, D. Munro \& L. Mann (Ed.), Heterogeneity in cross-cultural psychology (pp. 537-547). Lisse, Netherlands: Swets and Zeitlinger.

Tomkins, S.S. (1962). Affect, imagery, and consciousness (Vol. 1: The positive affects). New York: Springer.

Tomkins, S.S. (1963). Affect, imagery, and consciousness (Vol. 2: The negative affects). New York: Springer.

Ward, C. (2001). The A, B, Cs of Acculturation. In D. Matsumoto (Ed.), Handbook of Culture and Psychology (pp. 411-446). New York: Oxford University Press. 
Ch16.qxd 7/8/05 5:12 PM Page 402 